\title{
Modificaciones en la gestión del espectro: opciones y situación actual
}

\author{
Claudio Feijóo \\ CeDInt \\ Universidad Politécnica de Madrid \\ José Luis Gómez Barroso \\ Asunción Mochón \\ Dpto. Economía Aplicada e Historia Económica \\ Universidad Nacional de Educación a Distancia (UNED)
}

\section{Resumen}

El objetivo de este artículo es presentar las opciones que están considerándose (incluso ya aplicándose) para la reforma de los mecanismos de gestión del espectro radioeléctrico y evaluar su conveniencia y oportunidad. En concreto, se describen y valoran los tres cambios más profundos en este posible cambio de modelo: autorización del mercado secundario, utilización de la subasta para la asignación primaria y liberalización plena del uso del espectro. El proceso ya en marcha de introducción de estos cambios en España es también analizado.

Palabras clave: espectro radioeléctrico, recursos escasos, subasta, comercio secundario, neutralidad tecnológica.

Clasificación JEL: K23, L50, L96.

\section{Abstract}

The objective of this article is to present the reforms in the radioelectric spectrum management mechanisms that are currently being drafted (or that are even being applied) as well as to assess their advisability and timeliness. In particular, the article assesses the three deepest changes that are being considered: authorisation of the secondary market, usage of auctions for primary allocation and full liberalisation of spectrum usage. The changes under way in Spain are also analysed.

Keywords: radio electric spectrum, scarce resources, auctions, secondary market, technological neutrality.

JEL classification: K23, L50, L96.

\section{Introducción}

La regulación del espectro radioeléctrico está condicionada por dos supuestos: el espectro radioeléctrico es un recurso escaso (o, cuando menos, limitado) y es, además, un bien preciado que se utiliza en servicios esenciales para la sociedad: aplicaciones vinculadas a la seguridad, la defensa y la respuesta ante emergencias, telecomunicaciones y radiodifusión, transporte o investigación científica.

Con estos postulados, el espectro radioeléctrico es considerado un bien de dominio público por las administraciones de prácticamente todo el mundo. Y, en consecuencia, los procedimientos tradicionales de otorgamiento de los derechos para su 
uso han venido estando estrictamente reglamentados. Básicamente, tras planificar el empleo que se da a cada banda de frecuencias, especificando el servicio que puede utilizarla y la tecnología que debe emplearse, se concede el derecho de uso a determinados agentes mediante decisiones administrativas en las que se detallan minuciosamente las obligaciones del concesionario.

Mediante este sistema se solventan los problemas que pudieran aparecer debidos a interferencias. Además, y puesto que el primer paso del proceso, la planificación, se coordina en organismos supranacionales, se asegura una aceptable (no siempre total) compatibilidad global de los sistemas de comunicación que, adicionalmente, genera importantes economías de escala en el mercado de dispositivos electrónicos. Como contraprestación a los derechos de uso otorgados a los concesionarios de las licencias, estos suelen estar obligados al pago de un canon anual (fijo o dependiente de sus resultados) y, en algunas ocasiones, a cumplir otros compromisos (con frecuencia de despliegue de red o de nivel de cobertura).

Este modelo, mantenido inalterado durante décadas, está perdiendo vigencia. Las nuevas circunstancias, tanto tecnológicas como económicas, que concurren en los mercados hacen que el modelo tradicional aparezca como excesivamente complejo y falto de flexibilidad. Ello ha conducido a la aparición de una corriente de opinión favorable a la introducción de criterios de economía de mercado en la gestión del espectro radioeléctrico ${ }^{1}$. De hecho, numerosas autoridades nacionales encargadas de la gestión del espectro $^{2}$, la Comisión Europea ${ }^{3}$ o la UIT ${ }^{4}$ ya hace algún tiempo han publicado documentos en que se propugna la modificación de los actuales métodos de gestión del espectro y en que se defiende la inclusión de mecanismos de mercado. Más importante aún, la reforma del marco regulador es ya efectiva en varios países y en España se están dando los pasos hacia una primera reforma positiva y no meramente normativa.

El objetivo de este artículo es presentar las opciones que existen para la reforma de los mecanismos de gestión del espectro radioeléctrico y evaluar su conveniencia

\footnotetext{
${ }^{1}$ En realidad, es un «reforzamiento» y no una «aparición» de esta corriente de opinión. Nada menos que en 1959, ya Coase argumentaba que el espectro radioeléctrico debía ser considerado como cualquier otro factor de producción y que su valor, por tanto, debía ser determinado por el mercado; los problemas de interferencias deberían ser resueltos recurriendo a la legislación general sobre propiedad privada (COASE, 1959).

${ }^{2}$ Tómese como ejemplo en Europa el del regulador británico OFCOM. Véase el documento «Spectrum framework review: Implementation plan» publicado en 2005 (disponible en http://www.ofcom. org.uk/consult/condocs/sfrip/).

Por su parte, la FCC cita como primer objetivo en su página web: «advance spectrum reform by developing and implementing market-oriented allocation and assignment reform policies». Véase http://www.fcc.gov/spectrum/

3 «Policy aims to facilitate spectrum access across the EU through market mechanisms». Comunicación de la Comisión «i2010 - Una sociedad de la información europea para el crecimiento y el empleo» COM (2005) 229 final. Bruselas, 1 de junio de 2005.

${ }^{4}$ Véase, a modo de ejemplo, el documento de la UIT «Market mechanisms for spectrum management». Se trata de un documento que resume los resultados de la reunión con el mismo título convocada en Ginebra los días 22 y 23 de enero de 2007 (disponible en http://www.itu.int/osg/spu/stn/spectrum/workshop_proceedings/STN.MMSM-2007-PDF-E.pdf).
} 
y oportunidad. El trabajo se estructura en seis secciones. En el apartado 2 se analizan las razones que hacen conveniente un cambio de modelo y se resumen los rasgos más destacados de la transformación. Las secciones 3 a 5 describen y valoran los tres cambios más profundos que se están considerando: autorización del mercado secundario, utilización de la subasta para la asignación primaria y liberalización plena del uso del espectro. Los cambios en la gestión del espectro en España ocupan el apartado 6. Se finaliza con las conclusiones que se derivan de los análisis anteriores y con algunas recomendaciones personales para guiar el cambio.

\section{La necesidad de un cambio de modelo}

\subsection{Objetivos en la gestión del espectro}

El principal objetivo de todo mecanismo de gestión del espectro es (o debiera ser) alcanzar la mayor eficiencia posible en su uso. Este principio genérico de eficiencia puede desglosarse en tres dimensiones: eficiencia técnica, eficiencia económica y eficiencia social. Nótese que estas tres dimensiones, como se verá tras su definición, no son autónomas. Nótese también que estos conceptos se aplican a los mecanismos de asignación y gestión, y no al empleo que los agentes hagan del espectro que les ha sido concedido.

- La eficiencia técnica exige que el mayor número posible de frecuencias esté disponible para ser utilizado de manera efectiva en la prestación de servicios ${ }^{5}$. Esto implica que en el caso de existir varias tecnologías con que ofrecer un determinado servicio en las mismas condiciones, debe optarse por aquella que menos recursos (frecuencias) emplee. Adicionalmente, deben reducirse las bandas de guarda en la medida de lo técnicamente viable: es decir, sin que dicha reducción signifique que las interferencias entre distintas señales radioeléctricas hagan imposible un aprovechamiento real de determinadas bandas.

- La eficiencia económica sería máxima cuando, de la combinación de posibles usos del recurso (el espectro), se eligiera la válida para «producir» aquellos servicios que optimizaran la utilidad de los agentes presentes en el mercado (Morris, 2005). Debe así evitarse asignar espectro a servicios y/o tecnologías que no son las más demandadas por el mercado y deben retirarse los derechos de uso a aquellas que han quedado obsoletas.

- La eficiencia social implica que el espectro radioeléctrico debe emplearse en aquellos servicios o aplicaciones que maximicen el bienestar de la sociedad. En este sentido, es preciso, por ejemplo, destinar ciertas bandas del espectro a actividades como la investigación o la seguridad, actividades que serían descartadas si sólo se considerara la maximización del beneficio empresarial (o incluso la utilidad del consumidor) en el corto plazo.

${ }^{5}$ Lo que, en terminología económica, supondría situarse en la frontera de posibilidades de producción (de utilización del recurso). 
En todos los casos, el análisis debe ampliarse introduciendo una prospectiva de desarrollo futuro o, en otras palabras, optimizar una definición dinámica, y no meramente estática, de la eficiencia.

Cabe señalar además, como se había adelantado anteriormente, la interrelación existente entre las tres dimensiones. Por ejemplo, un incremento en la eficiencia técnica libera frecuencias, lo que posibilita la entrada de nuevos agentes o la aparición de nuevos servicios; el incremento de la competencia que resultaría se traduce en beneficios para los usuarios o para la sociedad en su conjunto. Incluso desde un punto de vista conceptual la eficiencia social podría entenderse como económica si se interpretan las situaciones descritas como fallos de mercado. No obstante todo lo anterior, la distinción resulta útil a efectos de estructuración del estudio.

\subsection{Características del nuevo escenario}

La introducción paulatina de mejoras y de innovaciones tanto en las tecnologías de manejo del espectro radioeléctrico como en las tecnologías que utilizan espectro radioeléctrico ha originado profundos cambios en los mercados. A su vez, los cambios en los mercados impulsan la investigación que, en un a modo de círculo virtuoso, impulsa nuevos cambios tecnológicos.

Sin que, por tanto, puedan establecerse causalidades entre unos y otros avances, sí puede al menos considerarse cuáles de estas transformaciones afectan a cada una de las tres dimensiones de la eficiencia en el uso del espectro:

- Respecto a la eficiencia técnica:

- Desarrollo de tecnologías que reducen la cantidad de espectro necesaria para enviar una misma cantidad de información. Un caso paradigmático y actual es el cambio del formato analógico a digital en la difusión terrestre de televisión.

- Introducción de técnicas de acceso dinámico al espectro (tales como, por ejemplo, las técnicas de «radio cognitiva») que permiten una muy flexible gestión y reutilización ${ }^{6}$ del espectro en cualquiera de sus dimensiones (frecuencia, tiempo, localización geográfica) (véase Nekovee, 2006).

- Aparición de tecnologías que utilizan un rango de espectro muy amplio con, al mismo tiempo, una muy baja emisión de potencia (tecnologías de banda ultra ancha), lo que causa un nivel de interferencia muy reducido y su posible «convivencia» con otros servicios que operen en la misma banda.

- Innovaciones con repercusión en la parte de acceso de las redes (redes en malla o mesh networks, femtoceldas) que permiten reutilización de frecuencias y un aumento de la eficiencia en su uso.

\footnotetext{
${ }^{6}$ Considérese que medidas de utilización real del espectro muestran que una parte considerable del mismo no se usa durante periodos de tiempo importantes (segundos, incluso minutos) (WEISS y JONDRAL, 2004).
} 
- Respecto a la eficiencia económica:

- Proliferación de servicios que requieren espectro radioeléctrico para su operación.

- Existencia de servicios que han quedado obsoletos y que emplean mucho más espectro del que sería necesario con otras alternativas tecnológicas o, en el caso extremo, que no utilizan en absoluto las bandas de frecuencia que tienen asignadas (y que, por tanto, deberían ser reasignadas).

- Creciente utilización de bandas que no precisan licencia de uso, lo que relativiza la necesidad de una planificación tan detallada. No sólo creciente sino exitosa: piénsese en WiFi o en Bluetooth.

- Rápida evolución de la tecnología (no siempre previsible), lo que dificulta la asignación a priori de bandas del espectro a unas tecnologías concretas, que pueden quedar rápidamente desfasadas.

- Respecto a la eficiencia social:

- Importancia estratégica de muchos servicios que necesitan espectro y que son considerados herramientas habilitadoras para el desarrollo de la sociedad de la información.

\subsection{Claves para un nuevo modelo}

Los nuevos modos de gestionar el espectro pretenden aumentar la flexibilidad y la transparencia, y también la rapidez de respuesta antes las innovaciones tecnológicas. La demanda real y el valor que el mercado concede a cada banda de frecuencias son nuevos criterios directores. Obviamente, todo cambio debería mejorar la eficiencia global en la utilización del recurso, siempre manteniendo ciertos requisitos técnicos irrenunciables.

Son tres las vías posibles de cambio. De abajo hacia arriba en el proceso de asignación de los derechos de uso del espectro:

- Modificaciones en las condiciones de la licencia asignada: flexibilización de algunas cláusulas pero, especialmente, autorización de la transmisión de los derechos (comercio secundario de espectro).

- Modificaciones en los mecanismos de asignación de las licencias: uso de la subasta.

- Modificaciones en la definición de las licencias: no ligar la licencia a unas tecnologías concretas (neutralidad tecnológica) o ni siquiera a unos servicios concretos (neutralidad de servicios).

Estas tres vías no constituyen diferentes alternativas. Se puede optar por varias de estas herramientas, y ello podría hacerse de manera simultánea o sucesiva. Sin 
embargo, tampoco son completamente independientes y, como veremos más adelante, la introducción de determinados cambios está casi inevitablemente asociada con otras modificaciones del modelo.

Analizamos a continuación las características de estas posibles reformas.

\section{Mercado secundario del espectro}

\subsection{Concepto}

La «comercialización del espectro» supone la subrogación, total o parcial, de los derechos que le han sido otorgados a un agente para hacer uso de un segmento del dominio público radioeléctrico.

La comercialización crea una nueva forma de acceder al espectro. Las compañías interesadas pueden conseguir los derechos de uso bien a través de la asignación primaria, si son seleccionados por la autoridad competente en materia de asignación de espectro, bien a través del mercado secundario, adquiriendo los derechos de uso a otro agente.

Los motivos que impulsarían a un operador con derechos de uso del dominio público radioeléctrico a decidir subrogarlos serán de índole económica: dicho operador recibe una remuneración por parte del agente que adquiere el derecho (de forma directa o indirecta, si la subrogación se vincula a otros acuerdos entre los dos intervinientes) o bien la transmisión supone una reducción de costes (por ejemplo, el ahorro del canon o tasa radioeléctrica anual).

Por su parte, los intereses del operador que consigue los derechos de uso del espectro pueden ser muy variados: entrar en un nuevo mercado, conseguir espectro adicional para mejorar la prestación de sus servicios, evitar la entrada de nuevos competidores, etc.

\subsection{Modalidades de cesión de los derechos de uso}

El Cuadro 1 establece una clasificación de los diferentes criterios con que se podría realizar la cesión de los derechos. 
CUADRO 1

MODALIDADES DE CESIÓN DE LOS DERECHOS DE USO

\begin{tabular}{|c|c|c|}
\hline Criterio & Modalidades & Particularidades \\
\hline \multirow{2}{*}{ Temporal } & Permanente & $\begin{array}{l}\text { La subrogación se establece por tiempo ilimitado, renun- } \\
\text { ciando el agente cedente al derecho de uso de forma defini- } \\
\text { tiva (cesión irrevocable) }\end{array}$ \\
\hline & Provisional & $\begin{array}{l}\text { La subrogación se establece por un período de tiempo deter- } \\
\text { minado en el acuerdo. }\end{array}$ \\
\hline \multirow{2}{*}{ Geográfico } & Total & $\begin{array}{l}\text { El agente cedente renuncia al derecho de uso del espectro en } \\
\text { todo el ámbito geográfico de la licencia }\end{array}$ \\
\hline & Regional & $\begin{array}{l}\text { El agente cedente y el cesionario comparten parte o la totali- } \\
\text { dad de las bandas de frecuencia en diferentes zonas geográ- } \\
\text { ficas }\end{array}$ \\
\hline \multirow{2}{*}{ Amplitud } & Completa & $\begin{array}{l}\text { El agente cesionario de los derechos de uso ha de subrogar- } \\
\text { se en los derechos y obligaciones del agente cedente }\end{array}$ \\
\hline & Parcial & $\begin{array}{l}\text { El agente cedente mantiene las obligaciones asumidas frente } \\
\text { a la Administración. Resulta preciso delimitar las obligacio- } \\
\text { nes que adquiere, en su caso, el agente cesionario }\end{array}$ \\
\hline \multirow{2}{*}{ Revocabilidad } & Revocable & $\begin{array}{l}\text { De acuerdo con las condiciones estipuladas, el agente ceden- } \\
\text { te puede revocar la cesión del derecho }\end{array}$ \\
\hline & Irrevocable & $\begin{array}{l}\text { El agente cedente renuncia de forma definitiva al derecho de } \\
\text { uso del espectro }\end{array}$ \\
\hline
\end{tabular}

FUENTE: Elaboración propia.

De permitirse en la práctica todas estas modalidades, los operadores tendrían a su disposición un abanico de opciones para elegir la que más conviniera a su estrategia comercial o a su situación particular.

Existe una posibilidad adicional. Si bien todas las categorías del Cuadro 1 implican la renuncia del operador cedente a todos o parte de sus derechos, en la llamada «compartición» de derechos, el espectro es utilizado por dos operadores sin que ello suponga menoscabo apreciable de las condiciones de uso del titular primero. Las condiciones para el uso compartido del espectro tienen su límite en la «interferencia soportable» o nivel máximo de ruido generado por el nuevo usuario que no perturba el servicio del propietario original de la banda. Esta modalidad se encuentra estrechamente ligada a las nuevas posibilidades que ofrecen los avances tecnológicos mencionados anteriormente, tales como la banda ultra ancha o las técnicas de radio cognitiva. 


\subsection{Ventajas e inconvenientes derivados de la comercialización del espectro}

El hecho de permitir la comercialización del espectro radioeléctrico puede traducirse en una serie de ventajas:

- Aumento de la eficiencia técnica en el uso del espectro: los agentes tendrán incentivos para realizar un uso más eficiente del espectro que les ha sido otorgado, minimizando la cantidad de espectro que precisan para la prestación de sus servicios y poniendo el resto en el mercado.

- Aumento de la competencia: el aumento de la eficiencia técnica supone indirectamente un aumento del espectro disponible y, consecuentemente, del número de oferentes de servicios.

- Fomento de la innovación: el establecimiento de un sistema de libre intercambio permite que las innovaciones tecnológicas lleguen antes al usuario final, ya que de un lado las empresas con derechos de uso del espectro tienen incentivos para introducir dichas innovaciones y, del otro, los nuevos operadores disponen de un mecanismo para entrar en los mercados mucho más veloz y flexible que la asignación primaria de frecuencias.

Frente a las potenciales ventajas esbozadas, el mercado secundario de espectro plantea problemas. Sería preciso considerar estos problemas de manera previa a la determinación del procedimiento para la cesión de espectro:

- Generación de incertidumbre: la cesión de espectro modifica las condiciones de competencia que se establecieron implícitamente en las asignaciones primarias de espectro, en la que se especificaba el número de licencias totales, lo que confería al mercado un cierto grado de estabilidad propicio para comprometer inversiones. No obstante, el efecto global sobre la inversión sería incierto puesto que los nuevos poseedores de derechos sólo podrían hacer su compra rentable en la medida en que llevaran a cabo inversiones.

- Distorsión de la competencia: determinados agentes pueden aprovechar la cesión de frecuencias para acumular espectro y lograr así posiciones de dominio en el mercado. Podrían además aparecer agentes que operaran con intereses meramente especulativos, encareciendo el precio del espectro sin aportar ningún valor a su gestión ni a su uso eficiente.

- Aparición de otros fallos de mercado: fallos asociados a los conceptos de eficiencia social y de eficiencia técnica (conflictos por interferencias, dificultades para el desarrollo de estándares), y también debidos a los costes de transacción. Aunque Valletti (2001), afirma que no sólo hay que considerar la importancia de estos fallos sino también si el regulador sería capaz de encontrar mejores soluciones para resolverlos que el propio mercado.

- Ineficiencia en el uso del recurso: una excesiva fragmentación del espectro podría originar un aumento notable de las frecuencias no disponibles por ser 
utilizadas como bandas de guarda. También podrían surgir problemas de coordinación internacional o meramente regional, si se ha producido una cesión de frecuencias limitada geográficamente.

\section{Modificación del método para realizar la asignación primaria: la subasta}

\subsection{Características de las subastas de espectro}

El concurso ha sido el mecanismo predominante (pero no único ${ }^{7}$ ) para la asignación primaria del espectro. El concurso sirve al regulador para determinar mediante criterios no sólo económicos (también sociales, incluso técnicos) qué solicitante es el más cualificado para utilizar el espectro.

Pese a lo anterior, la utilización de la subasta no es desconocida en el sector. Se han repartido licencias mediante subasta desde hace años y en un buen número de países. Hubo, sin embargo, un punto de inflexión en la consideración de las subastas como mecanismo de asignación de espectro. Fue en los años 2000 y 2001 cuando varios países europeos subastaron las licencias de tercera generación de comunicaciones móviles (UMTS). La altura a que llegaron las pujas ${ }^{8}$ hizo que las subastas de espectro llegaran incluso a los titulares de los medios de comunicación. Más recientemente, las subastas han vuelto a atraer la atención a raíz de la subasta de parte del dividendo digital ${ }^{9}$ en Estados Unidos o, con mucha menor incidencia mediática, en otros países como Alemania. De hecho, el reparto del dividendo digital promete un buen número de futuras (y próximas) subastas (véase al respecto, Gómez Barroso et al., 2011).

A raíz de las subastas de UMTS, comenzó a proliferar el estudio sobre modelos de subastas, tratando de alcanzar los mecanismos de asignación óptimos del espectro en términos de eficiencia y también en términos de maximización del beneficio (Banks et al., 2003; Milgrom, 2004; Plot y Salmon 2004). Y es que el diseño del

7 Los sorteos constituyen un método rápido, económico y transparente para seleccionar entre diversos solicitantes precalificados. Aunque los sorteos fueron empleados por la FCC hasta la década de los noventa, no son una solución eficiente, ya que el agraciado con la licencia puede no ser quien más la valora o quien ofrece el servicio y plan de despliegue más beneficiosos socialmente.

8 Medido por los ingresos obtenidos por el Estado, no todas las subastas fueron igualmente exitosas. En realidad, los resultados fueron muy diferentes de unos países a otros, oscilando entre los 20 euros per cápita de Suiza y los 650 euros per cápita obtenidos en la subasta del Reino Unido. Esta gran dispersión se debió, fundamentalmente, al deficiente diseño de la subasta en algunos países y a la secuencia temporal con la que se desarrollaron las subastas (KLEMPERER, 2002).

9 Espectro que queda «libre» tras la transición de la transmisión analógica a digital de la señal terrestre de televisión por ser la transmisión digital mucho más eficiente espectralmente. El tamaño de este dividendo tiene una base tecnológica pero es, fundamentalmente, una decisión política, pues prácticamente en todo el mundo una parte del dividendo posible se asigna a la emisión de nuevos canales de televisión. 
modelo de subasta condiciona por entero los resultados que se obtengan del proceso.

Cuando se trata de subastar múltiples lotes (como en el caso de las subastas de espectro), la primera y más importante de las decisiones que debe tomar la agencia encargada de la gestión del espectro es si la venta de los lotes se realiza de forma independiente la venta de los lotes de forma independiente o conjunta.

- Los principales tipos de subastas cuando las licencias se venden en subastas independientes son ${ }^{10}$ :

- Subastas secuenciales: Las licencias se venden de una en una en subastas separadas.

- Subastas simultáneas: Las licencias se venden al mismo tiempo en diferentes subastas y los postores pueden pujar por los lotes que deseen. En la subasta simultánea de múltiples rondas (Simultaneous Multiple-Round, SMR; también llamada Simultaneous Ascending Auction, SAA) los participantes realizan sus pujas y los precios más altos se toman como partida para la siguiente ronda; todas las subastas permanecen activas hasta que ninguno de los lotes recibe nuevas pujas.

- Si el vendedor opta por vender todas las licencias a la vez en una única subasta (especialmente cuando los lotes no son completamente homogéneos), el vendedor debe decidir cómo agrupar los lotes.

Es norma habitual permitir que los participantes realicen ellos mismos las combinaciones («paquetes») que deseen. Este tipo de subastas se denominan combinatorias. De entre ellas cabe destacar (Cramton et al., 2006):

- Subasta combinatoria iterativa (subasta ascendente de múltiples rondas): Al principio de cada ronda, los postores pujan por uno o más paquetes. El vendedor resuelve el problema de optimización (no obvio por la propia estructura de la subasta) que supone la determinación de las pujas que maximizan su ingreso (winner-determination problem), anuncia la asignación provisional y pide precios para la ronda siguiente. La subasta finaliza en el momento fijado al inicio o cuando no se realizan nuevas pujas.

- Subasta ascendente con apoderado (ascending proxy): Los participantes informan de sus valores a un apoderado (proxy). Este apoderado puja en nombre del postor en cada ronda. El vendedor considera todas las pujas (de cualquiera de las rondas) y elige la combinación de pujas más rentable para él (incluyendo no más de una puja o «un paquete» por postor). El proceso continúa hasta que no hay nuevas pujas.

${ }^{10}$ CRAMTON (2002) comenta las ventajas y desventajas de estos modelos de subastas, con pujas ascendentes o a sobre cerrado, en la asignación del espectro. 
- Subasta clock-proxy: Subasta híbrida con dos tramos: un primer tramo de múltiples rondas, y un segundo tramo correspondiente a una única puja a sobre cerrado. En el primer tramo, el vendedor anuncia unos precios y los postores declaran cuál sería su demanda para esos precios. Los precios se aumentan hasta que ninguno de los lotes tiene exceso de demanda. Es entonces cuando se pasa a la segunda parte en que los postores comunican sus preferencias a un apoderado (podría ser el propio vendedor).

De entre estos posibles formatos de subasta, las subastas combinatorias están especialmente indicadas si las licencias puedan ser, entre ellas, tanto sustitutivas como complementarias (existencia de sinergias). Las subastas combinatorias, que permiten a los participantes hacer pujas tanto por paquetes completos (all-or-nothing bids) como por licencias individuales, evitan el llamado «riesgo de agregación» ${ }^{11}$. Sin embargo, y pese a estas ventajas, su uso no está generalizado debido a su complejidad tanto para el subastador (en la solución del winner-determination problem) como para los postores (a la hora de comprender la dinámica de la subasta y realizar sus pujas).

\subsection{Comparación entre concurso y subasta}

Se analizan a continuación una serie de puntos que se traen a colación en todo debate entre los defensores de uno y otro método de asignación. Cabe indicar, no obstante, que las características del mercado en cuestión y, sobre todo, el diseño del proceso (sea subasta o concurso) son parámetros básicos que podrían alterar las conclusiones generales. Un concurso bien planeado es siempre preferible a una subasta deficiente y viceversa. Se trataría, pues, de analizar qué método es preferible, supuesto un diseño correcto de ambas opciones.

- Los concursos son acusados de ausencia de transparencia y de credibilidad. En efecto, con independencia del rigor de los criterios de evaluación, los procedimientos de selección contienen un elemento subjetivo, por lo que algunas veces son denominados «concursos de belleza» (beauty contest). Esta subjetividad suele despertar la sospecha de que los organismos encargados de tomar las decisiones pudieran no ejercer sus funciones de manera imparcial. En ocasiones, las decisiones son criticadas, y hasta impugnadas, lo que afecta a la credibilidad del proceso de concesión de licencias y, en general, a la credibilidad de la administración responsable.

\footnotetext{
${ }^{11}$ En presencia de sinergias, los participantes en una subasta se enfrentan al riesgo de agregación (aggregation risk o exposure problem) que se produciría si no obtienen el número mínimo de licencias deseado.
} 
Las subastas, por su parte, constituyen un medio transparente y objetivo para otorgar las licencias.

- Como argumento frente a la subasta, se indica que el concurso permite asignar el espectro a los operadores con mayores compromisos de inversión, lo que habría de repercutir en la extensión y la calidad de servicio o la creación de empleo.

Sin embargo, un procedimiento de precalificación apropiado (incluyendo criterios de cobertura y de calidad) garantiza que los adjudicatarios de la subasta cuenten con la capacidad técnica y financiera necesaria para iniciar la provisión de servicios de forma rápida.

- Los concursos rebajan las barreras de entrada al mercado para los operadores, pues no exigen un pago previo para comenzar a operar (o, de hacerlo, es generalmente mucho menor). Consecuentemente, los operadores pueden realizar una distribución racional de la inversión y disponen inicialmente de mayores recursos económicos para desplegar infraestructuras y desarrollar servicios.

La misma tesis puede darse la vuelta en el caso de la subasta: el desembolso necesario para ganar una subasta también pueden considerarse un incentivo para la rápida expansión de las redes. De hecho, esta es la única manera con la que el ganador de una subasta puede recuperar la inversión (coste hundido) que representa el precio pagado por la licencia.

- Ligado con el argumento anterior, se objeta que los operadores vencedores de una subasta imputarán el coste de la licencia directamente a los servicios finales que ofrecen. $\mathrm{O}$, en otras palabras, que los consumidores pagarán unos precios más altos. No está demostrado que este comportamiento de los operadores sea posible. Dependería, obviamente, de la estructura y las condiciones de competencia en el mercado. En su estudio, Bauer (2003) concluye que «aunque hay ligeros indicios de que el precio de las licencias se traslada a los precios, no existe evidencia estadísticamente significativa de que este efecto sea duradero».

- El concurso crea rentas económicas asociadas al poder de mercado que se crea al limitar ex ante el número de agentes presentes en el mercado (Hazlett, 2003). Nada cambia en el caso de la subasta, salvo que se permita transferir la licencia obtenida.

- Un argumento que se utiliza con frecuencia en favor de las subastas de espectro es que proporcionan la mayor renta posible por la utilización de un recurso público. Esta renta podría destinarse a planes sociales del propio sector (por ejemplo, financiación del servicio universal).

Sin embargo, no es este un argumento con validez absoluta. Aparte, claro está, que se deben evitar en la subasta comportamientos colusivos (en que los postores acuerden de antemano el precio) lo cierto es que los ingresos dependen del modelo de subasta y de las valoraciones y estrategias de los postores. Además, el resultado de la subasta es siempre una incógnita por lo que una administración con «aversión al riesgo» preferiría conocer con mayor certeza lo que 
probablemente ingresará y optaría por el concurso (o quizá por una subasta con precio de reserva). El tipo de servicio es también en estos casos un parámetro de decisión: en servicios con poca demanda, podría darse el caso de que el número de postores fuera menor que el de derechos subastados.

- Pueden aparecer factores exógenos (una depresión económica, una nueva tecnología) que vuelvan difíciles de cumplir los compromisos adquiridos en el momento de la adjudicación de un concurso.

No es infrecuente que en las subastas también se incorporen exigencias de despliegue o de servicio. De ser así, nada cambia con respecto al concurso. Sí es cierto que la relajación o supresión de las obligaciones ligadas a la concesión de la licencia va unida a la utilización de la subasta pues deja al concurso sin parámetros de decisión.

\section{Liberalización del uso del espectro}

Como se indicó al inicio, la asignación de una determinada licencia de uso del espectro conlleva obligaciones tradicionalmente muy estrictas: servicio que se va a proveer, tecnología concreta que debe ser empleada, limitaciones de la potencia de emisión, ámbito geográfico en el que se puede radiar y ancho de banda de cada canal. La liberalización del uso del espectro permite que algunas o todas las condiciones anteriores puedan ser relajadas u omitidas, de forma que los agentes que disfrutan de los derechos sobre el dominio público radioeléctrico sean los que tengan capacidad para decidir el uso y la tecnología que van a implantar.

La neutralidad tecnológica (libre elección de la tecnología con que ofrecer el servicio) no presenta excesivos problemas, tanto desde el punto de vista conceptual, como en la práctica, especialmente si las alternativas tecnológicas, como suele ser el caso, no presentan características de emisión radicalmente diferentes.

Más compleja (por más «revolucionaria») es la configuración de un modelo regulador del espectro es que se permita además la neutralidad de servicios (libre elección del uso que se da a la banda). Existen fundamentalmente tres modelos para conseguir esta «plena» liberalización del espectro:

- En el modelo de «propiedad privada» se definen el contenido y la duración ${ }^{12}$ de los derechos de uso y se realiza una asignación primaria de los mismos. Una vez las licencias han sido otorgadas, el agente poseedor del derecho tiene com-

\footnotetext{
12 Aunque sería posible que el Estado transmitiera efectivamente la propiedad del derecho sobre el espectro, esta no parece la opción más probable (véanse defensas de esta opción en SPILLER y CARDILLI, 1999, o en WHITE, 2000). Sería esperable que lo hiciera por un número de años determinado (aun con posibilidad de renovación) lo que, jurídicamente, acercaría la figura más a la posesión o a un derecho de uso y disfrute.
} 
pleta libertad de actuación ${ }^{13}$, con las únicas restricciones de las normas superiores (legislación mercantil o de defensa de la competencia).

- En el modelo «comunitario» (modelo de los commons), el recurso se considera de uso compartido y, por tanto, no existen derechos de uso particulares definidos (Werbach, 2004). Para operar no es necesario siquiera disponer de una licencia formal. Es una modalidad ya aplicada en ciertas bandas de uso libre, como las que utiliza WiFi.

- Como alternativa intermedia entre los dos modelos anteriores, Faulhaber y Farber (2003) proponen un modelo de propiedad privada eludible en condiciones de no interferencia. En este régimen, el espectro se podría comprar y vender sin más restricción en el uso que la referida a la potencia de la emisión. Pero este derecho de uso sería preferente y no absoluto pues cualquier otro agente tendría el derecho a emitir en esa banda siempre que cumpliera con la condición de no interferencia. Sería un mecanismo similar a la compartición descrita en la sección 4.2 pero sin necesidad de acuerdo previo. Se establecería así de facto un régimen de uso que se podría denominar «comunitario con condiciones».

La liberalización de uso del espectro se enfrenta, en cualquiera de sus modalidades, a los problemas que el método tradicional de asignación minuciosa de servicios había resuelto: compatibilidad global de los sistemas de comunicación, creación de estándares (con las consiguientes economías de escala en el mercado de dispositivos electrónicos) y prevención de interferencias.

El problema de las interferencias es particularmente agudo en el caso de considerar el recurso comunitario, y muy especialmente si el uso es intensivo y el recurso se vuelve escaso («tragedy of the commons»). Los otros dos modelos resuelven (al menos parcialmente) el problema pero a costa de incrementar (puede que extraordinariamente) los costes de transacción.

Aunque los actores privados pasan a ser los protagonistas en estos modelos, sigue existiendo un papel para los organismos de gestión del espectro: vigilancia de que los usuarios cumplen las condiciones de protección del dominio público radioeléctrico, control de los límites de exposición para la protección sanitaria, evaluación de riesgos por emisiones radioeléctricas, control de que las negociaciones entre agentes se desarrollan en un marco de competencia. Como regla general, la intervención pública ex ante se minimiza y aumenta el control ex post.

${ }^{13}$ Lo que incluiría el derecho a comerciar con la licencia. Como se dijo en la sección 2, las reformas en la gestión del espectro no son completamente independientes y no parece concebible un marco en que se introduzca la liberalización de uso pero no el mercado secundario. 


\section{Los primeros pasos hacia el cambio de modelo en España}

El nuevo paquete regulador europeo de las comunicaciones electrónicas, el tercero tras la liberalización del sector, publicado en el Diario Oficial de la Unión Europea el 18 de diciembre de 2009 incorpora como una de las reformas destacables «reforzar la flexibilidad en la gestión del espectro y en el acceso al mismo, al amparo de autorizaciones neutras con respecto a la tecnología y los servicios».

Ya antes, en septiembre, se habían aprobado dos normas que incorporaban en la práctica tales principios. Tanto la Directiva 2009/114/CE ${ }^{14}$, que abre la posibilidad de que la banda de $900 \mathrm{MHz}$ sea utilizada por tecnologías distintas a GSM, como la Decisión 2009/766/CE ${ }^{15}$, que hace extensible esta modificación también a la banda de $1.800 \mathrm{MHz}$, incorporan el principio de neutralidad tecnológica. Las bandas de 900 y $1.800 \mathrm{MHz}$ ya no están así vinculadas exclusivamente a la tecnología GSM, lo que implica que, siempre que no se causen interferencias a otros usuarios de estas bandas, podrán ser utilizadas por otras tecnologías que optimicen su uso.

La transposición al ordenamiento jurídico español de dichas normas está prevista que se haga aprovechando la publicación de la Ley de Economía Sostenible, aún en trámite legislativo en el momento de la escritura de este artículo. A la espera de que este trámite se cumpla, las acciones necesarias para llevar a la práctica la medida están ya en marcha. La Directiva habilita a los Estados miembros a modificar o reconsiderar los derechos de uso del espectro (es decir, a alterar los actuales títulos habilitantes) si es que, como consecuencia de su aplicación, la competencia en los mercados pudiera verse afectada. La Directiva también indica que cualquier decisión en este sentido habría de ir precedida de una consulta pública. Es la razón por la que entre el 15 de junio y el 15 de julio de 2010 el Ministerio de Industria, Turismo y Comercio tuvo abierta la «Consulta pública sobre actuaciones en materia de espectro radioeléctrico: refarming en las bandas de 900 y $1.800 \mathrm{MHz}$, dividendo digital y banda de 2,6 GHz».

Como se observa, el título de la consulta menciona otras bandas de frecuencia. La banda de 790-862 MHz es parte del dividendo digital y durante $2009^{16}$ el Gobierno anunció que, de acuerdo con lo recomendado por la Comisión Europea, se destinaría para servicios de acceso móvil de banda ancha. Estas frecuencias, junto con los $190 \mathrm{MHz}$ libres de la banda de 2,6 GHz (la otra banda citada en la consulta),

\footnotetext{
${ }^{14}$ Directiva 2009/114/CE del Parlamento Europeo y del Consejo de 16 de septiembre de 2009 por la que se modifica la Directiva 87/372/CEE del Consejo relativa a las bandas de frecuencia a reservar para la introducción coordinada de comunicaciones móviles terrestres digitales celulares públicas paneuropeas en la Comunidad.

15 Decisión 2009/766/CE de la Comisión, de 16 de octubre de 2009, relativa a la armonización de las bandas de frecuencias de $900 \mathrm{MHz}$ y $1800 \mathrm{MHz}$ para los sistemas terrenales capaces de prestar servicios paneuropeos de comunicaciones electrónicas en la Comunidad.

16 Decisión que se concretó en el Real decreto 365/2010, de 26 de marzo, por el que se regula la asignación de los múltiples de la televisión digital terrestre tras el cese de las emisiones de televisión terrestre con tecnología analógica. La banda de $800 \mathrm{MHz}$ debe quedar libre para poder ser asignada a sus nuevos usos antes de finalizar el año 2014.
} 
deberían permitir a los operadores disfrutar del espectro suficiente para sostener la creciente demanda de comunicaciones de banda ancha y desplegar los sistemas de cuarta generación de comunicaciones móviles.

El Real Decreto que se debe publicar, tras considerar las respuestas a la consulta pública, detallará los aspectos concretos de esta recolocación de frecuencias, en concreto, cómo se ven alterados los actuales títulos de uso. Dejando a un lado los detalles concretos sobre la posible liberación de bloques de frecuencia o su reasignación entre los operadores con red ahora establecidos, desde el punto de vista de la gestión del espectro son tres son los aspectos que interesan.

El primero, aún sin respuesta, es qué procedimiento se utilizará en la licitación de las concesiones demaniales que se otorgarán en la banda de frecuencias de 800 $\mathrm{MHz}$ (seis de ámbito estatal) y en la de $2,6 \mathrm{GHz}$ (en la que se ha previsto que algunas tengan ámbitos territoriales inferiores al estatal).

El segundo es el alcance de la neutralidad en el uso de todas las bandas afectadas por el Real decreto. Es seguro que se impondrá neutralidad tecnológica, si consideramos las comunicaciones móviles (sean de datos o de voz) como un único servicio. No queda claro en el borrador de Real decreto si se trata de una neutralidad de servicios absoluta, en cuyo caso se debería admitir que se utilizara parte de este espectro para, por ejemplo, emitir más canales de televisión digital terrestre.

El último se refiere a la posible transferencia de títulos habilitantes o a la cesión de derechos de uso del dominio público radioeléctrico, es decir, al comercio secundario de espectro. Parece que va a permitirse en todas las bandas citadas anteriormente pero también en otras destinadas a las comunicaciones móviles como son la banda de $2.100 \mathrm{MHz}$ y la de 3,5 GHz (3,4-3,6 GHz).

\section{Conclusiones}

Que el espectro radioeléctrico está necesitado de un modelo de gestión nuevo (o al menos de un modelo renovado) es una aseveración sobre la que existe consenso tanto entre responsables públicos como entre los actores con intereses en el mercado o entre investigadores.

Considerando el análisis que se ha realizado en este capítulo (y especialmente las ventajas e inconvenientes presentados en cada caso), se propone una serie de pautas para la introducción de reformas en los mecanismos de gestión del espectro:

- La reforma ha de ser necesariamente progresiva en profundidad (qué medidas), alcance (qué bandas de frecuencia) y calendario. Con independencia de su oportunidad, no es viable una liberalización plena e inmediata de todo el espectro comercialmente útil.

- Ello implica una sustitución paulatina de la regulación ex ante del esquema tradicional por una regulación flexibilizada (o totalmente ex post). El periodo de convivencia entre diferentes modelos será largo o incluso indefinido, pues la 
liberalización plena no tiene por qué ser necesariamente el objetivo último. Pueden existir servicios o mercados para los que, por su inmadurez, trascendencia u otra razón, sea preferible mantener el modelo tradicional de gestión.

- Las modificaciones en la política de gestión del espectro tendrán efectos significativos en el mercado sólo a medio plazo. Por ello es determinante diseñar un escenario que, partiendo de la situación actual, identifique los objetivos perseguidos (fundamentalmente en términos de eficiencia). Deberá monitorizarse periódicamente el grado de consecución de los objetivos, resaltando las mejores prácticas e introduciendo las medidas correctoras necesarias.

- El paso previo a cualquier reforma ha de ser la evaluación, asumiendo la gran complejidad que ello conlleva, de la ocupación actual de cada banda. Dicho análisis condicionará en gran parte las medidas concretas que puedan aplicarse. En particular servirá para seleccionar bandas piloto en las que poner a prueba modelos alternativos.

- Dadas las diferentes características que presentan las distintas bandas puede que no resulte viable la aplicación de un único modelo de gestión del espectro. Sería necesario, por tanto, realizar un análisis banda por banda que determine el modelo básico de gestión que resulte más adecuado para cada una de ellas (de entre los posibles de «regulación tradicional», «introducción paulatina de mecanismos de mercado» y «modelo liberalizado»), e identifique los distintos servicios a que se puede dedicar su uso.

- La introducción de mecanismos de mercado es compatible con la fijación de unos criterios de eficiencia técnica y/o económica utilizables en el control que deben ejercer los organismos de administración y gestión del espectro.

- Podrían así establecerse unos umbrales de utilización del recurso de forma que los agentes que operaran por debajo del nivel mínimo de eficiencia fueran penalizados o, en último término y ante casos de uso ineficiente continuado, pudieran ver revocados sus derechos de uso. Este tipo de herramienta sería además útil en la lucha contra prácticas anticompetitivas tales como la acumulación de espectro con el fin de establecer barreras de entrada al mercado.

- Es necesario que todos los procesos relacionados con la gestión del espectro sean públicos. Esto implica la publicación de la estrategia global del organismo encargado del espectro, el mantenimiento actualizado del registro de frecuencias (incluyendo información relevante sobre las asignaciones: frecuencia, ancho de banda, duración, fecha en la que se ha realizado la asignación y fecha de expiración, servicio, posibilidad de comercio secundario, precio) e información sobre el grado de utilización de cada banda, sobre los criterios de eficiencia y sobre los procedimientos de resolución de disputas. En concreto, el registro de frecuencias es determinante para una efectiva introducción del comercio del espectro (véase Bykowsky, 2003).

Las primeras reformas que se están afrontando en España (en realidad en Europa) cumplen, en términos generales, la mayor parte de estos requisitos. Los merca- 
dos de comunicaciones móviles parecen lo suficientemente maduros como para aprovechar las oportunidades que la nueva regulación brinda de manera que se generen beneficios y se eviten los efectos no deseados que pudieran darse. Si la experiencia, como así debiera ser, es positiva no cabe duda de que lo que ahora se resalta como excepción a medio plazo será muy probablemente la norma.

\section{Referencias bibliográficas}

[1] BANKS, J.; OLSON, M.; PORTER, D.; RASSENTI, S. y SMITH, V. (2003): «Theory, experimental and the Federal Communications Commission spectrum auctions», Journal of Economic Behaviour and Organization., vol. 51, n. ${ }^{\circ}$ 3, pp. 303-350.

[2] BAUER, J. M. (2003): «Impact of license fees on the prices of mobile voice services», Telecommunications Policy, vol. 27, n. ${ }^{\circ}$ 5-6, pp. 417-434.

[3] BYKOWSKY, M. (2003): «A secondary market for the trading of spectrum: promoting market liquidity», Telecommunications Policy, vol. 27, n. ${ }^{\circ}$ 7, pp. 533-541.

[4] COASE, R. (1959): «The Federal Communication Commission», The Journal of Law \& Economics, vol. 2, pp. 1-40.

[5] CRAMTON, P. (2002): «Spectrum auctions» en Cave, M.; Majumdar, S. y Vogelsang, I., eds., Handbook of Telecommunications Economics, pp. 605-639. Elsevier Science B.V. (Amsterdam).

[6] CRAMTON, P.; SHOHAM, Y. y STEINBERG, R. (2006): Combinatorial auctions. The MIT Press (Cambridge).

[7] FALCH, M. y TADAYONI, R. (2004): «Economic versus technical approaches to frequency management», Telecommunications Policy, vol. 28, n. ${ }^{\circ}$ 2, pp. 197-211.

[8] FAULHABER, G. R. y Farber, D. J. (2003): «Spectrum management: property rights, markets, and the Commons», en Cranor, L.F. y Wildman, S.S., eds., Rethinking rights and regulations: Institutional responses to new communication technologies, pp. 193226. The MIT Press (Cambridge).

[9] GÓMEZ BARROSO, J. L.; MOCHÓN, A.; SÁEZ, Y. y FEIJÓO, C. (2011): «The importance of the size of the digital dividend when digital dividend spectrum is auctioned», International Journal of Mobile Communications, vol. 9, n. ${ }^{\circ}$ 1, pp. 57-75.

[10] HAZLETT, T. W. (2003): «Liberalizing US spectrum allocation», Telecommunications Policy, vol. 27, n. ${ }^{\circ}$, pp. 485-499.

[11] KLEMPERER, P. (2002): «How (not) to run auctions: The European 3G telecom auctions», European Economic Review, vol. 46, n. . 4-5, pp. 829-845.

[12] MILGROM, P. (2004): Putting auction theory to work. Cambridge University Press (Cambridge)

[13] MORRIS, A. C. (2005): «Spectrum auctions: Distortionary input tax or efficient revenue instrument?», Telecommunications Policy, vol. 29, n. ${ }^{\circ}$ 9-10, pp. 687-709.

[14] NEKOVEE, M. (2006): «Dynamic spectrum access - concepts and future architectures», BT Technology Journal, vol. 24, n. ${ }^{\circ}$ 2, pp. 111-116.

[15] PLOT, C. R. y SALMON, T. C. (2004): «The simultaneous ascending auction: dynamics of price adjustment in experiments and in the UK $3 \mathrm{G}$ spectrum auction», Journal of Economic Behaviour and Organization, vol. 53, n. ${ }^{\circ} 3$, pp. 353-383. 
[16] SPILLER, P. T. y CARDILLI, C. (1999): «Towards a property rights approach to communication spectrum», Yale Journal on Regulation, vol. 16, n. ${ }^{\circ}$ 1, pp. 53-84.

[17] VALETTI, T. M. (2001): «Spectrum trading», Telecommunications Policy, vol. 25, n. ${ }^{\circ}$ 10/11, pp. 655-670.

[18] WEISS, T. A. y JONDRAL, F. K. (2004): «Spectrum pooling: An innovative strategy for the enhancement of spectrum efficiency», IEEE. Communications Magazine, vol. 42, . $^{\circ} 3$, pp. S8-S14.

[19] WERBACH, K. (2004): «Supercommons: Toward a unified theory of wireless communication», Texas Law Review; vol. 82, n. ${ }^{\circ}$ 4, pp. 863-973.

[20] WHITE, L. J. (2000): «Propertyzing' the electromagnetic spectrum: why it's important, and how to begin», Media Law \& Policy, vol. 9, n. ${ }^{\circ}$ 1, pp. 29-30. 